\title{
Cluster spin glass behavior in geometrically frustrated $\mathrm{Zn}_{3} \mathrm{~V}_{3} \mathrm{O}_{8}$
}

\author{
T. Chakrabarty* \\ Department of Physics, IIT Bombay, Powai, Mumbai 400076, India \\ A. V. Mahajanđ and S. Kundu \\ Department of Physics, IIT Bombay, Powai, Mumbai 400076, India
}

\begin{abstract}
We report the bulk magnetic properties of a yet unexplored vanadium-based multi-valenced spinel system, $\mathrm{Zn}_{3} \mathrm{~V}_{3} \mathrm{O}_{8}$. A Curie-Weiss fit of our $d c$ magnetic susceptibility $\chi(T)$ data in the temperature region of 140-300 K yields a Curie constant $C=0.75 \mathrm{~cm}^{3} \mathrm{~K} /$ mole $\mathrm{V}, \theta_{C W}=-370 \mathrm{~K}$. We have observed a splitting between the zero field cooled ZFC and field cooled FC susceptibility curves below a temperature $T_{i r r}$ of about $6.3 \mathrm{~K}$. The value of the 'frustration parameter' $\left(\frac{\left|\theta_{\mathrm{CW}}\right|}{T_{\mathrm{N}}} \sim 100\right)$ suggests that the system is strongly frustrated. From the $a c$ susceptibility measurements we find a logarithmic variation of freezing temperature $\left(T_{f}\right)$ with frequency $\nu$ attesting to the formation of a spin glass below $T_{f}$. However, the value of the characteristic frequency obtained from the Vogel-Fulcher fit suggests that the ground state is closer to a cluster glass rather than a conventional spin glass. We explored further consequences of the spin glass behavior and observed aging phenomena and memory effect (both in ZFC and FC). We found that a positive temperature cycle erases the memory, as predicted by the hierarchical model. From the heat capacity $C_{P}$ data, a hump-like anomaly was observed in $C_{P} / T$ at about $3.75 \mathrm{~K}$. Below this temperature the magnetic heat capacity shows a nearly linear dependence with $T$ which is consistent with the formation of a spin glass state below $T_{f}$ in $\mathrm{Zn}_{3} \mathrm{~V}_{3} \mathrm{O}_{8}$.
\end{abstract}

PACS numbers: 75.50.Lk, 75.40.Gb, 75.50.Ee

\section{INTRODUCTION}

A great deal of research has been done on the ground state properties of geometrically frustrated antiferromagnets during the last decade $\stackrel{1,2}{2}$ Cubic spinels $\left(\mathrm{AB}_{2} \mathrm{O}_{4}\right)$ and pyrochlores $\left(\mathrm{A}_{2} \mathrm{~B}_{2} \mathrm{O}_{7}\right)$ with magnetic ions at the $\mathrm{B}$ sites are important in this respect due to the formation of a geometrically frustrated corner-shared tetrahedral network among themselves. $\mathrm{Na}_{4} \mathrm{Ir}_{3} \mathrm{O}_{8}$ is a famous compound of the spinel family found in recent times where $\mathrm{Ir}$ and $\mathrm{Na}$ at the B-sites have distinct positions and the Ir ions form corner shared triangles in three dimensions. This has been dubbed as the hyperkagome lattice and is proposed to have a quantum spin liquid ground state. In some cases where there is deviation from the frustrated geometry, the resulting non-uniform interaction can lead to the formation of a spin glass SG state which is an exotic example of a nonergodic state marked by several other physical properties such as slow dynamics, nonexponential decay, aging effect, memory effect, etc $\stackrel{3,4}{.}$. In pyrochlores and spinels, spin glass behavior is often observed where the requirement of non uniform interaction can be readily satisfied. The nonequilibrium spin glass state arises from frustration due to competing magnetic interactions among the spins as well as disorder capable of pinning the spins. In the last few years, spin-glass-like nonequilibrium dynamics and timedependent behavior have been observed in several magnetic systems, where the basic building blocks responsible for this "glassy" behavior are spin clusters or a bigger spin entities rather than atomic spins ${ }^{5,6}$ Till now this sort of behavior has been prominently observed in manganites,$\frac{7.8}{1}$ cobaltites,,$-\frac{9}{-11}$ intermetallic alloys,,$\frac{6,12,13}{-16}$ other oxides $\stackrel{14,15}{12}$ as also in magnetic nanoparticles 16 where the frozen state is not the conventional spin glass but is rather referred to as a cluster glass since the basic building block is bigger than the simple atomic spins. Till date very few vanadium-based oxides have been placed in this category.

We were in the quest for new spinel compounds with magnetic ions at the B-sites, with the intention of unraveling novel magnetic properties arising due to geometric frustration. In this paper, we report our studies on the yet unexplored system $\mathrm{Zn}_{3} \mathrm{~V}_{3} \mathrm{O}_{8}$ via magnetization and heat capacity. Here, although there are magnetic $\mathrm{V}$ atoms at the $\mathrm{B}$ sites, the network is diluted due to the presence of nonmagnetic $\mathrm{Zn}$, a third of which reside at the B site. Our magnetization data are consistent with the presence of two $\mathrm{V}^{3+}(S=1)$ and one $\mathrm{V}^{4+}(S=1 / 2)$ ions per formula unit. A large, negative, Curie-Weiss temperature $\theta_{C W}=-370 \mathrm{~K}$ is found which is indicative of strong antiferromagnetic (AF) interactions. A difference between the zero field cooled (ZFC) and field cooled (FC) susceptibilities is observed below about $6.3 \mathrm{~K}$. The heat capacity also has a low temperature anomaly. Further, $a c$ susceptibility measurements corroborate the formation of a spin glass state, more specifically, a cluster spin glass. To study the dynamics of this glassy state below the freezing temperature $\left(T_{f}\right)$, we carried out further experiments and observed magnetic relaxation, memory and aging phenomena which are thought to be typical characteristics of spin glass dynamics. During the study of memory effects, we observed that a positive heat cycle erases the previous memory and initializes the relaxation again as prescribed by the hierarchical model $\stackrel{16,17}{\underline{1}}$ 
Table I: Atomic positions in $\mathrm{Zn}_{3} \mathrm{~V}_{3} \mathrm{O}_{8}$

\begin{tabular}{|c|c|c|c|c|}
\hline \multirow{2}{*}{ Atoms } & \multicolumn{3}{|c|}{ Co-ordinates } & Occupancy \\
\cline { 2 - 5 } & $\mathrm{x} / \mathrm{a}$ & $\mathrm{y} / \mathrm{b}$ & $\mathrm{z} / \mathrm{c}$ & \\
\hline $\mathrm{Zn} 1(8 \mathrm{a})$ & 0.000 & 0.000 & 0.000 & 0.985 \\
\hline $\mathrm{Zn} 2(16 \mathrm{~d})$ & 0.625 & 0.625 & 0.625 & 0.225 \\
\hline $\mathrm{V}(16 \mathrm{~d})$ & 0.625 & 0.625 & 0.625 & 0.775 \\
\hline O1(32e) & 0.734 & 0.734 & 0.734 & 1.000 \\
\hline
\end{tabular}

\section{EXPERIMENTAL DETAILS AND STRUC- TURAL INFORMATION}

$\mathrm{Zn}_{3} \mathrm{~V}_{3} \mathrm{O}_{8}$ was prepared by standard solid state reaction methods. First we prepared $\mathrm{V}_{2} \mathrm{O}_{3}$ by reducing $\mathrm{V}_{2} \mathrm{O}_{5}$ (Aldrich-99.99\%) in hydrogen atmosphere at $650^{\circ} \mathrm{C}$ for 16 hours and $\mathrm{VO}_{2}$ by mixing $\mathrm{V}_{2} \mathrm{O}_{3}$ with $\mathrm{V}_{2} \mathrm{O}_{5}$ in a $1: 1$ molar ratio, pelletizing and firing in dynamical vacuum (better than $10^{-5}$ mbar) at $800^{\circ} \mathrm{C}$ for 24 hours. In the first step we pelletized a mixture of $\mathrm{ZnO}$ (Aldrich $99.99 \%$ purity, dried at $140^{\circ} \mathrm{C}$ to remove moisture), $\mathrm{V}_{2} \mathrm{O}_{3}$, and $\mathrm{VO}_{2}$ which was then sealed in an evacuated quartz tube. This was then fired at $650^{\circ} \mathrm{C}$ for 24 hours. After regrinding and repelletizing, the sample was again fired in an evacuated quartz tube at $850^{\circ} \mathrm{C}$. X-ray diffraction (xrd) patterns were collected with a PANalytical x-ray diffractometer using $\mathrm{Cu} \mathrm{K} \mathrm{K}_{\alpha}$ radiation $(\lambda=1.54182)$. From the Rietveld refinement of the xrd pattern (see Fig. 11) the lattice parameter was found to be $8.402 \AA$ in the $F d-3 m$ space group. The results of the refinements are shown in Table I below. The goodness of the Rietveld refinement is defined by the following parameters. $\mathrm{R}_{p}=3.94 \%, \mathrm{R}_{w p}$ $=5.42 \%, \mathrm{R}_{\text {exp }}=2.33 \%$, and $\chi^{2}=5.39$.

In the structure of $\mathrm{Zn}_{3} \mathrm{~V}_{3} \mathrm{O}_{8}$ the B-sites are shared by zinc $\left(\mathrm{Zn}^{2+}\right.$ has an ionic radius of $\left.0.74 \AA\right)$ and vanadium (both $\mathrm{V}^{4+}$ and $\mathrm{V}^{3+}$ ) in a 1 : 3 ratio to form a cornershared tetrahedral network [see Fig. 2]. The $\mathrm{V}^{4+}$ ion $(S=1 / 2$ and ionic radius $0.58 \AA)$ differs slightly in size from the $\mathrm{V}^{3+}$ ion $(S=1$ and ionic radius $0.64 \AA)$. Due to this difference in ionic size the corner-shared tetrahedral network may be distorted and due to the difference in the value of spin the various $3 d-3 d$ nearest neighbour magnetic interactions will likely be different. In addition to this, due to the random/statistical occupation of the $\mathrm{B}$ sites by $\mathrm{Zn}$ and $\mathrm{V}$, there are going to be missing magnetic ions in the triangular network. Therefore, $\mathrm{Zn}_{3} \mathrm{~V}_{3} \mathrm{O}_{8}$ can be regarded as a bond-disordered geometrically frustrated antiferromagnet, as for $\mathrm{LiCrMnO}_{4}{ }^{18}$ This disruption/dilution of the corner-shared tetrahedral network serves as an additional source of disorder and is likely to lead to relieving of frustration and an eventual spin glass/frozen state.

The temperature dependence of magnetization $M$, its relaxation, memory effect, aging phenomenon and several other dynamic properties were measured in the temperature range 2-300 K using a SQUID VSM from Quantum Design. The temperature dependence of heat capacity

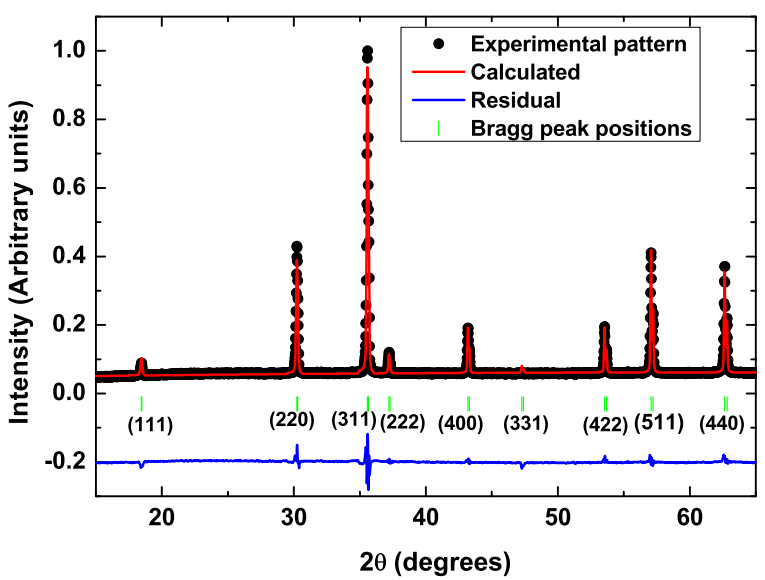

Figure 1: Powder diffraction pattern of $\mathrm{Zn}_{3} \mathrm{~V}_{3} \mathrm{O}_{8}$ is shown along with its Bragg peak positions; The black points are the experimental data, the red solid line is the calculated refinement pattern, the blue solid line is the residual data and the green markers are the Bragg peak positions for $F d-3 m-s$ space group.

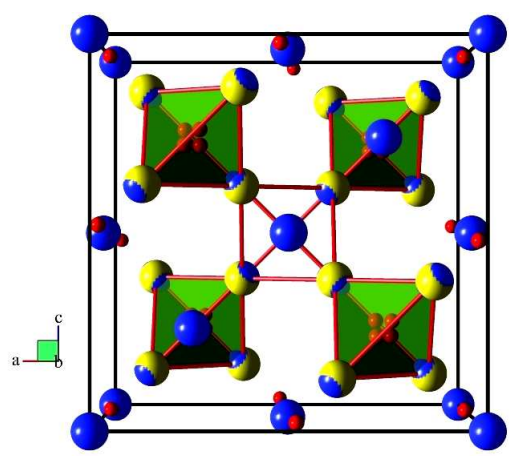

Figure 2: The unit cell of $\mathrm{Zn}_{3} \mathrm{~V}_{3} \mathrm{O}_{8}$. The $\mathrm{Zn}^{2+}, \mathrm{V}^{4+} / \mathrm{V}^{3+}$, $\mathrm{O}^{2-}$ ions are shown in blue, yellow, and red color, respectively. The B-sites form a corner-shared tetrahedral network consisting of $\mathrm{V}^{3+}, \mathrm{V}^{4+}$, and $\mathrm{Zn}^{2+}$ ions.

has also been measured in the temperature range of 2$250 \mathrm{~K}$ using the heat capacity attachment of a Quantum Design PPMS.

\section{RESULTS AND DISCUSSIONS}

\section{A. Magnetic measurementsthe \\ 1. dc magnetization and ac susceptibility}

The reciprocal of the susceptibility is plotted with temperature in Fig. 3. From the Curie-Weiss fit $\chi(T)=$ $\chi_{0}+C /\left(T-\theta_{\mathrm{CW}}\right)$ in the range $140-300 \mathrm{~K}$, we get the $T$-independent susceptibility $\chi_{0}=4.66 \times 10^{-4} \mathrm{~cm}^{3} / \mathrm{mole}$ $\mathrm{V}$, the Curie constant $C=0.75 \mathrm{~cm}^{3} \mathrm{~K} /$ mole $\mathrm{V}$, and the 


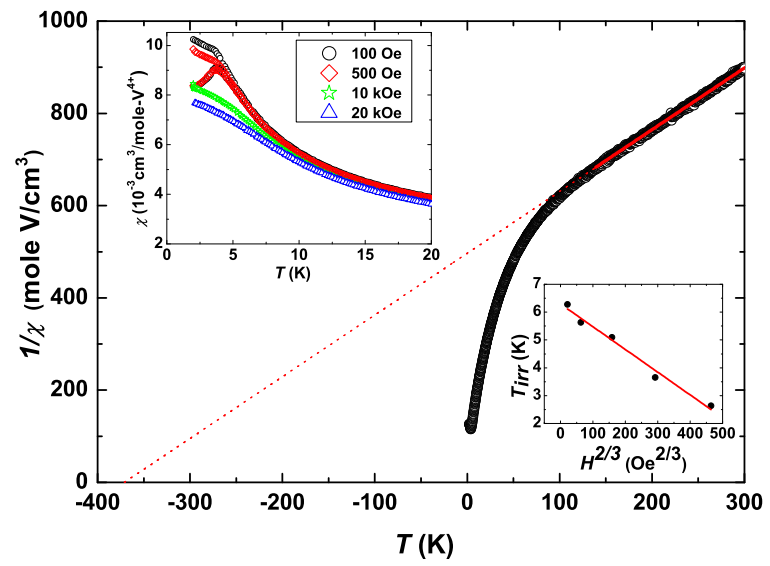

Figure 3: Temperature dependence of inverse susceptibility $1 /\left(\chi-\chi_{0}\right)$ is shown for $H=100$ Oe. The red line shows the Curie-Weiss fit in the temperature range of $140-300 \mathrm{~K}$ and the dotted line is its extrapolation. The left inset shows the bifurcation between the ZFC and FC curves for various fields. The right inset shows the de Almeida-Thouless line, plotted as $T_{i r r}$ vs $H^{2 / 3}$.

Curie-Weiss temperature $\theta_{\mathrm{CW}}=-370 \mathrm{~K}$. From $\chi_{0}=$ $4.66 \times 10^{-4} \mathrm{~cm}^{3} /$ mole- $\mathrm{V}$ we obtain the Van Vleck susceptibility $\chi_{\mathrm{VV}}=\chi_{0}-\chi_{\text {core }}=6.19 \times 10^{-4} \mathrm{~cm}^{3} /$ mole V where $\chi_{\text {core }}$ is the core diamagnetic susceptibility equal to $-1.53 \times 10^{-4} \mathrm{~cm}^{3} /$ mole formula unit 19 The value of the Curie constant for a $S=1 / 2$ system (with $g=2$ ) is expected to be $0.375 \mathrm{~cm}^{3} \mathrm{~K} /$ mole and $1 \mathrm{~cm}^{3} \mathrm{~K} / \mathrm{mole}$ for $S=1$. The Curie constant obtained from the fit (i.e., $C=0.792 \mathrm{~cm}^{3} \mathrm{~K} /$ mole $\mathrm{V}$ ) is consistent with having two $S=1$ and one $S=1 / 2$ magnetic ion per formula unit. In low fields, a difference betwen the ZFC and FC susceptibilities, suggestive of a spin glass transition, is seen below a $T_{i r r}$ of about $6.3 \mathrm{~K}$. The ZFC and FC curves coalesce in fields of $20 \mathrm{kOe}$. A large frustration parameter $\frac{\left|\theta_{\mathrm{CW}}\right|}{T_{\mathrm{N}}} \sim 100$ suggests that strong frustration is present in the sytem $\stackrel{20}{-}$ It is found that $T_{i r r}$ follows the $H^{2 / 3}$ law, which has been observed in many spin glass systems such as $\mathrm{Nd}_{5} \mathrm{Ge}_{3}\left(\operatorname{Ref}^{\underline{6}}\right), \mathrm{Nd}_{2} \mathrm{AgIn}_{3}\left(\operatorname{Ref}^{21}\right)$, and $\mathrm{U}_{2} \operatorname{IrSi}_{3}\left(\operatorname{Ref}^{22}\right)$.

Fig. 4 shows the temperature variations of the in-phase component of the ac magnetic susceptibility $\left(\chi^{\prime}(T, \nu)\right)$ measured between 3 and $5 \mathrm{~K}$ in the frequency range 17 $\leq \omega / 2 \pi \leq 967 \mathrm{~Hz}$. The $\chi^{\prime}(T, \nu)$ curve displays a peak at $T_{f} \sim 3.75 \mathrm{~K}$ and it shifts towards higher temperatures as the frequency $\nu$ changes from $17 \mathrm{~Hz}$ to $967 \mathrm{~Hz}$. The out of phase component of ac susceptibility $\chi^{\prime \prime}(T, \nu)$ also shows a peak at $3.75 \mathrm{~K}$ which however hardly shifts with a change in frequency (see Fig. 5). At higher temperatures, above the freezing temperature $T_{f}, \chi^{\prime \prime}(T)$ is nearly equal to zero while below $T_{f}$ it has a non zero value. Such behavior is characteristic of the SG transition and allows us to distinguish the SG compounds from the disordered AF systems, in which $\chi^{\prime \prime}(T)$ is constant and remains zero

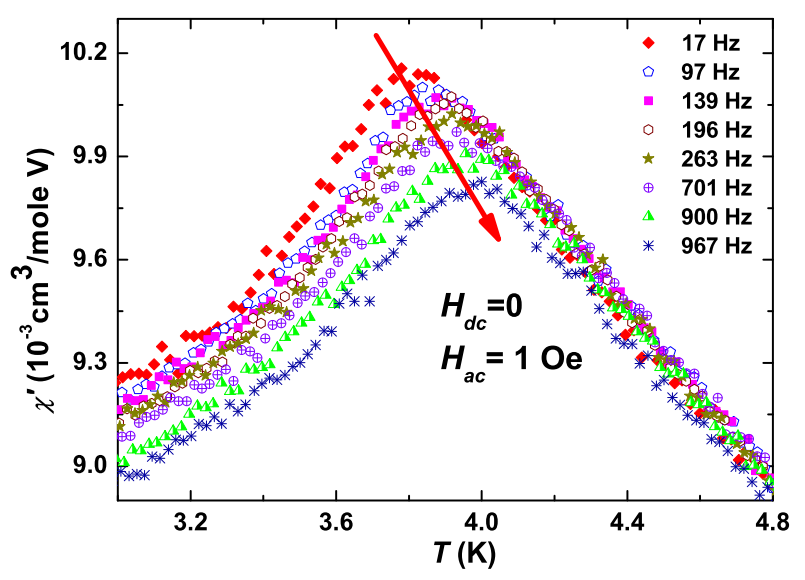

Figure 4: Temperature dependence of the real part of ac susceptibility $\left(\chi^{\prime}\right)$, measured at different frequencies with zero external $d c$ magnetic field and an $a c$ field of 1 Oe. The red arrow points to the shift in the spin-glass temperature $\left(T_{f}\right)$ with increase in frequency.

even below the transition temperature $\stackrel{23}{2}-25^{25}$ In Fig. 6, we depict $\chi^{\prime \prime}(T)$, normalized to the value at $17 \mathrm{~Hz}$, as a function of frequency for $\mathrm{Zn}_{3} \mathrm{~V}_{3} \mathrm{O}_{8}$, as an example. At $4.8 \mathrm{~K}$, i.e., above $T_{f}$, the variation of $\nu$ over two decades does not influence $\chi^{\prime}$ in a noticeable way. Below $T_{f}$, in the SG state, the $\chi^{\prime}$ exhibits a logarithmic frequency dependence. This kind of frequency dependence has been predicted theoretically for a short-range Ising $\mathrm{SG}^{26}$, and it has been observed for several other SG systems 9.27 . Note that at $3.6 \mathrm{~K}$, closer to $T_{f}$, the system is more sensitive to the variation of frequency than at $2.5 \mathrm{~K}$, which is "deeper" into the frozen state.

The $a c$ measurements at various frequencies reveal that the position of the maximum in the $\chi^{\prime}(T)$ curve $\left(T_{f}\right)$, moves toward higher temperatures and the magnitude of $\chi^{\prime}$ decreases with increasing frequency. Such behavior is expected for an SG system. Now, we will try to determine the category of $\mathrm{SG}$ to which $\mathrm{Zn}_{3} \mathrm{~V}_{3} \mathrm{O}_{8}$ belongs. As a raw measure of this frequency dependence, we have calculated the relative shift of the spin freezing temperature $\left(\delta T_{f}=\Delta T_{f} / T_{f} \Delta \log \nu\right) \underline{\underline{24}}$ and it comes out to be 0.028 . This value of $\delta T_{f}$ indicates that the sensitivity to the frequency is larger than that for canonical spin glasses such as $\mathrm{CuMn}\left(\delta T_{f}=0.005\right)($ Ref $\stackrel{28}{ }$ ) and AuMn $\left(\delta T_{f}=0.0045\right)\left(\operatorname{Ref}^{24}\right)$. It is in fact intermediate between the value of canonical spin glass system and superparamagnets ( for noninteracting ideal superparamagnetic systems such as holmium borate $(\alpha-$ $\left.\left.\left[\mathrm{Ho}_{2} \mathrm{O}_{3}\left(\mathrm{~B}_{2} \mathrm{O}_{3}\right)\right]\right), \delta T_{f} \sim 0.28\right)^{28}$ where the spin glass states appear due to the interaction between the randomly distributed magnetic clusters; however it is close to that typical for cluster glass systems. . The sensitivity to frequency strongly depends on the interaction between the particles or the magnetic clusters. In the case of mag- 


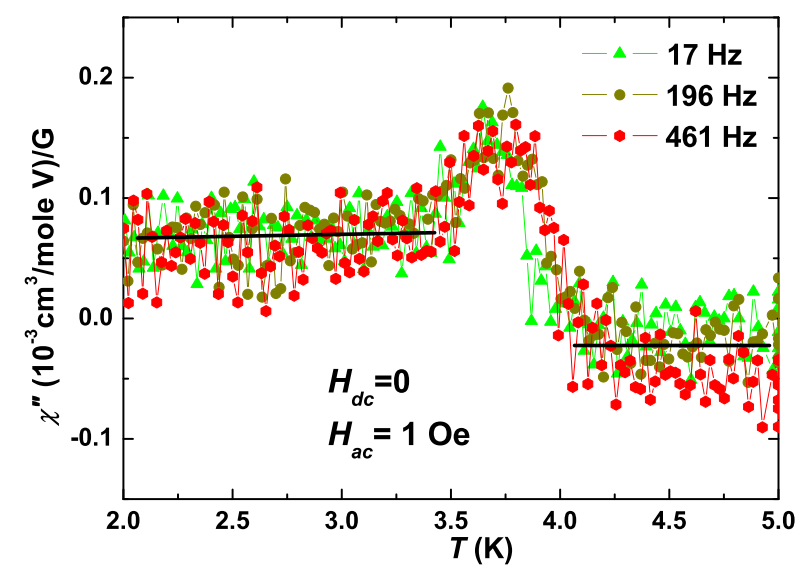

Figure 5: Temperature dependence of the out-of-phase part of $a c$ susceptibility $\left(\chi^{\prime \prime}\right)$, measured at different frequencies with zero external $d c$ magnetic field and an $a c$ field of amplitude 1 Oe.

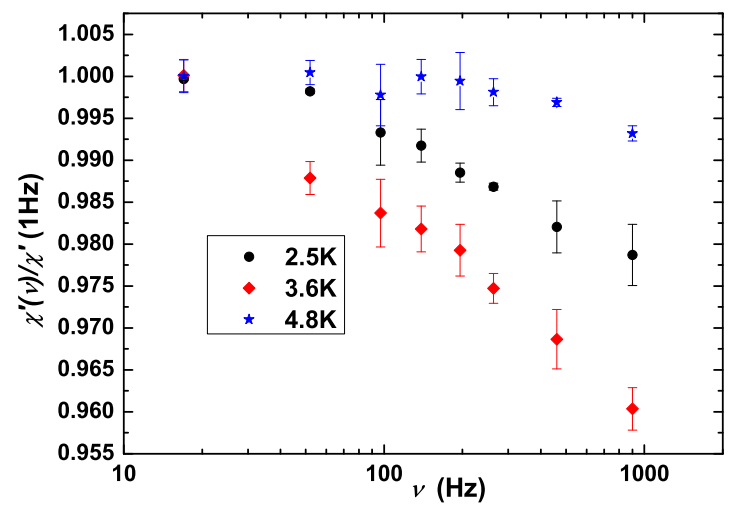

Figure 6: Normalized real part of $\chi^{\prime}(T)$ for $\mathrm{Zn}_{3} \mathrm{~V}_{3} \mathrm{O}_{8}$ as a function of frequency for temperatures above and below $T_{f}$. The measurements were carried out in an $a c$ field of amplitude 1 Oe and at zero $d c$ field.

netic clusters the interactions between the particles are weak and hence the sensitivity is strong. On the other hand, in a normal ferromagnetic and antiferromagnetic systems, the interaction between the magnetic atoms is strong and sufficiently large frequencies (usually $\mathrm{MHz}$ or $\mathrm{GHz}$ ) are needed to see any significant amount of shift in the frequency-dependent $a c$ susceptibility curves $\stackrel{9,28}{\underline{9}}$

In Fig. 7 we have shown the fit to the conventional expression of the critical slowing down of the relaxation times, $\tau / \tau_{0}=\left(T_{f} / T_{g}-1\right)^{-z \nu}$. In this expression, the characteristic time $\tau$ represents the dynamical fluctuation time scale and corresponds to the observation time $\left(t_{o b s}=1 / \omega=1 / 2 \pi \nu\right), \tau_{0}$ is the microscopic relaxation time, $T_{f}$ is the freezing temperature at a specific obser-

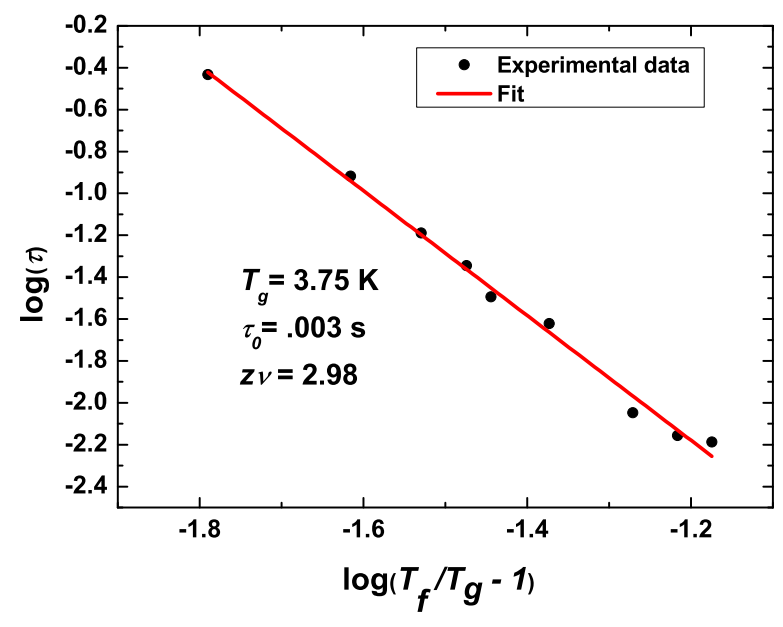

Figure 7: The best fit of spin freezing temperatures $\left(T_{f}\right)$ to the equation $\tau / \tau_{0}=\left(T_{f} / T_{g}-1\right)^{-z \nu}$

vation time, $T_{g}$ is the spin glass transition temperature which is equivalent to $T_{f}$ as $\nu \rightarrow 0$, and $z \nu$ is the dynamical exponent. The best fit is obtained for $T_{g} \approx 3.75$ $\mathrm{K}, z \nu \approx 2.98$ and $\tau_{0} \approx 3 \times 10^{-3} \mathrm{~s}$. For a conventional spin glass system the $\tau_{0}$ value lies between $10^{-10}$ and $10^{-13} \mathrm{~s}$ (Ref. ${ }^{6.29}$ ) and $z \nu$ ranges from 4 to 13 . The $\tau_{0}$ value obtained for $\mathrm{Zn}_{3} \mathrm{~V}_{3} \mathrm{O}_{8}$ is much higher than that for conventional spin glasses; in fact it is close to the values obtained in reentrant spin glass (RSG) systems and some other SG systems like $\mathrm{LaCo}_{0.5} \mathrm{Ni}_{0.5} \mathrm{O}_{3}$ (Ref $\left.\stackrel{14}{\underline{14}}\right), \mathrm{BiFeO}_{3}$ $\left(\operatorname{Ref} \underline{30}^{3}\right.$ ), and $\mathrm{LaMn}_{0.5} \mathrm{Fe}_{0.5} \mathrm{O}_{3}$ (Ref $\underline{31}$ ) where $\tau_{0}$ is close to $10^{-5} \mathrm{~S}$. Such a high value for $\tau_{0}$ indicates that the spin flipping takes place in a rather slow manner in $\mathrm{Zn}_{3} \mathrm{~V}_{3} \mathrm{O}_{8}$. The value of $z \nu$ is also less than the range for usual spin glasses.

In fig. 8 we have fitted $T_{f}$ with the empirical Vogel-Fulcher law, $\omega=\omega_{0} \exp \left[-E_{a} / k_{B}\left(T_{f}-T_{0}\right)\right]$, where $\omega_{0}$ is the characteristic frequency, $\omega$ is the angular frequency $(\omega=2 \pi \nu), E_{a}$ and $T_{0}$ are the activation energy and Vogel-Fulcher temperature, respectively. The best fit, shown in fig. 8, is obtained for $\omega_{0}=0.29 \times 10^{5} \mathrm{~Hz}$, $E_{a} / k_{B}=0.36 \mathrm{~K}$, and $T_{0}=3.76 \mathrm{~K}$. The characteristic frequency obtained from the fitting is much lower than that of conventional spin glass systems, which is about $10^{13}$ $\mathrm{rad} / \mathrm{s} \stackrel{22,32}{ }$ Such a low value of characteristic frequency is associated with RSG systems like $\mathrm{Ni}_{2} \mathrm{Mn}_{1.36} \mathrm{Sn}_{0.64} \stackrel{33}{\underline{3}}$ These observations clearly suggest that the spin glass state in $\mathrm{Zn}_{3} \mathrm{~V}_{3} \mathrm{O}_{8}$ is not atomic in origin; rather it is related to clusters of atoms and hence we would identify it as a cluster glass.

\section{Relaxation}

To study the dynamics of the glassy state, we measured the ZFC magnetization relaxation (see fig. 9) using the 


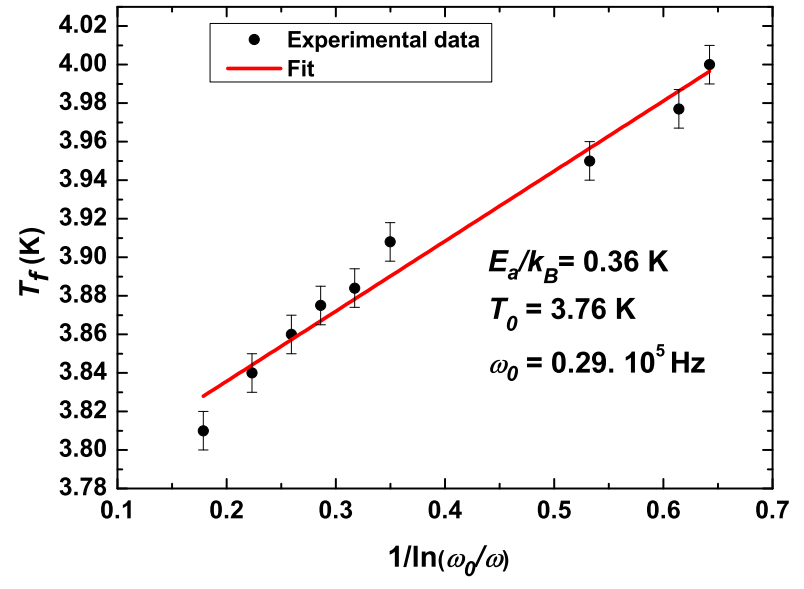

Figure 8: The plot of $T_{f}$ versus $1 / \ln \left(\omega_{0} / \omega\right)$ fitted with the Vogel-Fulcher law.

following protocol: the sample was cooled in zero field (ZFC) from well above $T_{f}$ to the measuring temperature. Once the measurement temperature was reached, a field of 100 Oe was applied and the magnetization $M_{Z F C}(t)$ was then recorded with time. We can clearly see that the ZFC magnetization does not saturate even after 2 h. This is because, in the glassy state, the moments are randomly frozen and it takes a long time for the field to turn those spins along the field direction. We have fitted the time dependence with the standard stretched exponential expression given below,

$M_{t}(H)=M_{0}(H)+\left[M_{\infty}(H)-M_{0}(H)\right]\left[1-\exp \left\{-(t / \tau)^{\alpha}\right\}\right]$

In this formula, $\tau$ is the characteristic relaxation time and $\alpha$ is the stretching parameter, which ranges between 0 and 1 . Here $M_{0}$ and $M_{\infty}$ are the magnetization values at $t \sim 0$ and $t \sim \infty$, respectively. The best fit is obtained for using $M_{\infty} / M_{0}=1.07$ and $1.05, \tau=1402 \mathrm{~s}$ and $627 \mathrm{~s}, \alpha=0.46$ and 0.45 for $T=1.8 \mathrm{~K}$ and $3 \mathrm{~K}$ respectively. Note that the growth of the magnetization is slower at $1.8 \mathrm{~K}$ compared to $3 \mathrm{~K}$ since at $1.8 \mathrm{~K}$ the system is deeper in the frozen state. However, no significant relaxation is observed in the $\mathrm{FC}$ case (not shown in Fig. 9). The value of $\tau$ obtained for $\mathrm{Zn}_{3} \mathrm{~V}_{3} \mathrm{O}_{8}$ is a bit smaller compared to those obtained in other spin glass systems like $\mathrm{Nd}_{5} \mathrm{Ge}_{3}\left(\operatorname{Ref}^{6}{ }^{6}\right)$ and $\mathrm{U}_{2} \mathrm{PdSi}_{3}\left(\operatorname{Ref}^{34}\right)$. The reason is that the value of $\tau$ depends on how deep into the frozen state the measurement has been carried out. In the above mentioned systems the $T_{f}$ is much higher (30 $\mathrm{K}$ for $\mathrm{Nd}_{5} \mathrm{Ge}_{3}(\operatorname{Ref} \underline{6})$ and $13.5 \mathrm{~K}$ for $\mathrm{U}_{2} \mathrm{PdSi}_{3}(\operatorname{Ref} .34)$ ) compared to that in $\mathrm{Zn}_{3} \mathrm{~V}_{3} \mathrm{O}_{8}$. So, when the measurements are carried out at $12 \mathrm{~K}$ (for $\mathrm{Nd}_{5} \mathrm{Ge}_{3}$ ) or at $2 \mathrm{~K}$, $5 \mathrm{~K}$ (for $\mathrm{U}_{2} \mathrm{PdSi}_{3}$ ) the system is already deep into the frozen state while for $\mathrm{Zn}_{3} \mathrm{~V}_{3} \mathrm{O}_{8}$, even at $1.8 \mathrm{~K}$ the system is not that deep into the frozen state compared to those

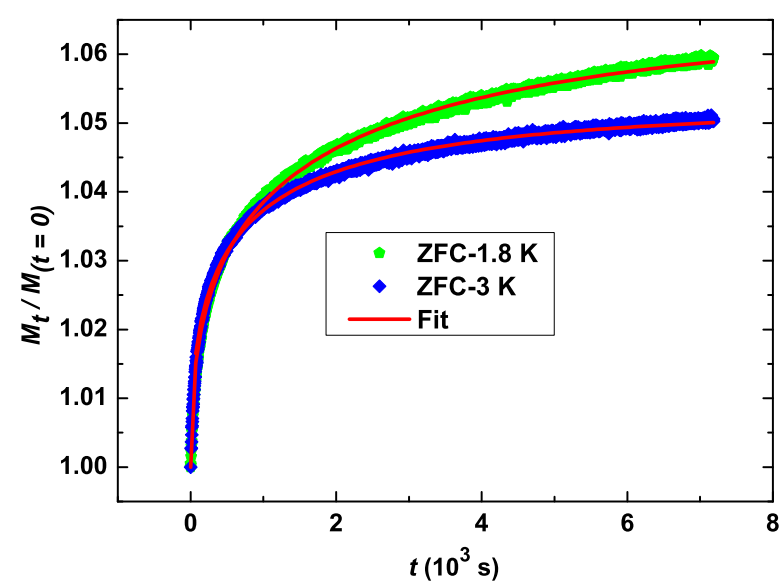

Figure 9: ZFC magnetization relaxation (normalised with respect to the magnetization at $t=0$ ) measured at $T=1.8$ $\mathrm{K}$ (green pentagon) and $3 \mathrm{~K}$ (blue diamond), $H=100$ Oe. The solid lines represent the fit with the stretched exponential expression given in text.

two systems. Indeed, the value of $\tau$ for $\mathrm{Zn}_{3} \mathrm{~V}_{3} \mathrm{O}_{8}\left(T_{f}=\right.$ $3.75 \mathrm{~K}$ ) at $1.8 \mathrm{~K}$ is $1402 \mathrm{~s}$ which is similar to what is obtained for $\mathrm{U}_{2} \mathrm{PdSi}_{3}\left(T_{f}=13.5 \mathrm{~K}\right)$ at $10 \mathrm{~K}(1411 \mathrm{~s}) \underline{34}$

We have also measured the isothermal remanent magnetization $\left(M_{I R M}\right)$ of $\mathrm{Zn}_{3} \mathrm{~V}_{3} \mathrm{O}_{8}$ to explore the metastable behavior of the glassy state at temperatures below the spin glass transition temperature (see Fig. 10). For this, first we cooled the sample in the ZFC mode from $150 \mathrm{~K}$ to the desired temperature, then a field of $40 \mathrm{kOe}$ was applied for $300 \mathrm{~s}$ and then the applied field was switched off. The magnetization was then recorded as a function of time for 1 hour. The decay of the remnant magnetization with time is significantly slow in the spin glass state. It is natural that the decay of $M_{I R M}$ is slower at lower temperatures (where the system is deeper into the frozen state) and gets faster as one gets closer to $T_{f}$. This indicates that the application of a field below $T_{f}$ causes the system to go to a metastable and irreversible state $\underline{6,22,34}$ As expected, above $T_{f} M_{I R M}$ is independent of time. Isothermal remnant magnetization $\left(M_{I R M}\right)$ data could also be fit using eqn.1 and the fit gives similar parameters as obtained from the $\mathrm{ZFC}$ magnetic relaxation data.

\section{Aging}

The nonergodic metastable state of $\mathrm{Zn}_{3} \mathrm{~V}_{3} \mathrm{O}_{8}$ is further verified by experiments manifesting the aging phenomena. To observe the aging effects, thermoremanent magnetization (TRM) was measured using the following protocol; the sample was first cooled in a field of $200 \mathrm{Oe}$ from a temperature well above $T_{f}$ to a stop temperature, $T_{s}=3.2 \mathrm{~K}$ (below $T_{f}$ ) where the system was allowed for 


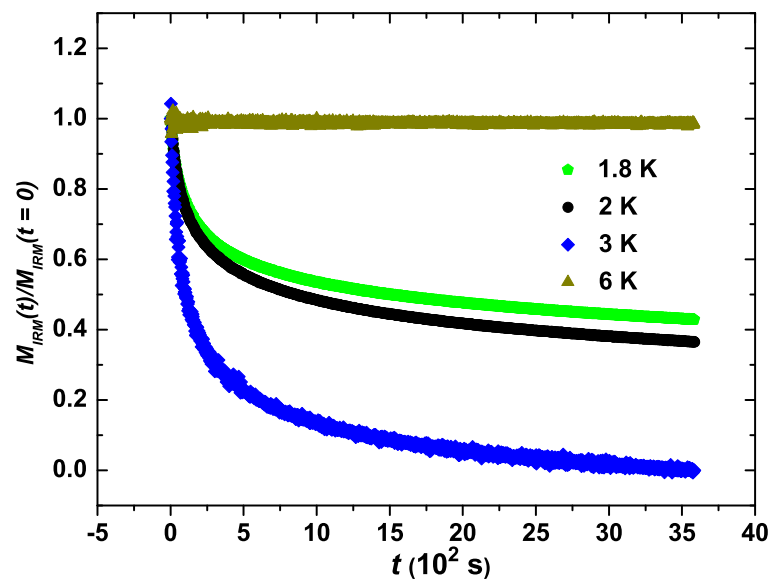

Figure 10: Isothermal remanent magnetisation normalised with respect to the value at $t=0$ is plotted as a function of time at $1.8 \mathrm{~K}$ (green pentagon), $2 \mathrm{~K}$ (black circle), $3 \mathrm{~K}$ (blue diamond) and $6 \mathrm{~K}$ (dark yellow triangle).

a stop time $t_{s 1}$ without changing the field. Then, the magnetic field was switched off and a second stop time $t_{s 2}$ was provided. The magnetic field was then set back to the original value (200 Oe) and the sample was immediately cooled down to the lowest temperature $(1.8 \mathrm{~K})$. Subsequently, the field was switched off and the magnetization was measured as a function of temperature during the heating. The measurement was performed for two different values of $t_{s 1}$, namely 5 and $5400 \mathrm{~s}$. The second stop time $t_{s 2}$ was kept constant $(5400 \mathrm{~s})$ for both the measurements. The salient feature of this measurement is that the two curves bifurcate at a temperature (3.66 $\mathrm{K}$ ) slightly below $T_{f}$ (see fig. 11), indicating the influence of the aging on the spin glass state. Similar behavior has been also seen in $\mathrm{La}_{0.95} \mathrm{Sr}_{0.05} \mathrm{CoO}_{3}$ (Ref $\left.{ }^{29}\right), \mathrm{Nd}_{5} \mathrm{Ge}_{3}$ (Ref. ${ }^{6}$ ), and $\operatorname{Ag}(\mathrm{Mn})$ (Ref. ${ }^{35}$ ) spin glass systems.

Fig 12 shows the growth of the magnetization data as a function of time, in the metastable state. The sample was cooled to $2.5 \mathrm{~K}$ in the ZFC mode and a field of 200 Oe was applied after a waiting time $t$. The magnetization was then measured as a function of time. As we can see, the magnetization growth is slower for larger waiting time, which again points that the metastability associated with the low temperature magnetic state.

\section{Memory effect}

To observe the existence of a memory effect ${ }^{16}$ in the $d c$ magnetization we have measured the FC magnetization using the following protocol. The magnetization was recorded during cooling of the sample at 500 Oe from 100 $\mathrm{K}$ down to $2 \mathrm{~K}$ at a constant cooling rate of $1 \mathrm{~K} / \mathrm{min}$. The cooling process was interrupted at $2.7 \mathrm{~K}$ and $2.2 \mathrm{~K}$

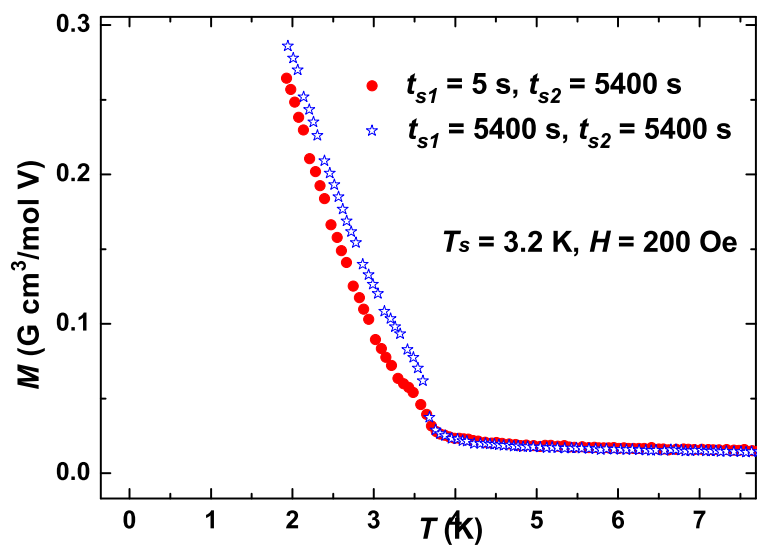

Figure 11: Thermoremanent magnetization (TRM) versus temperature for $T_{s}=3.2 \mathrm{~K}, H=200$ Oe after using different stopping times during cooling.

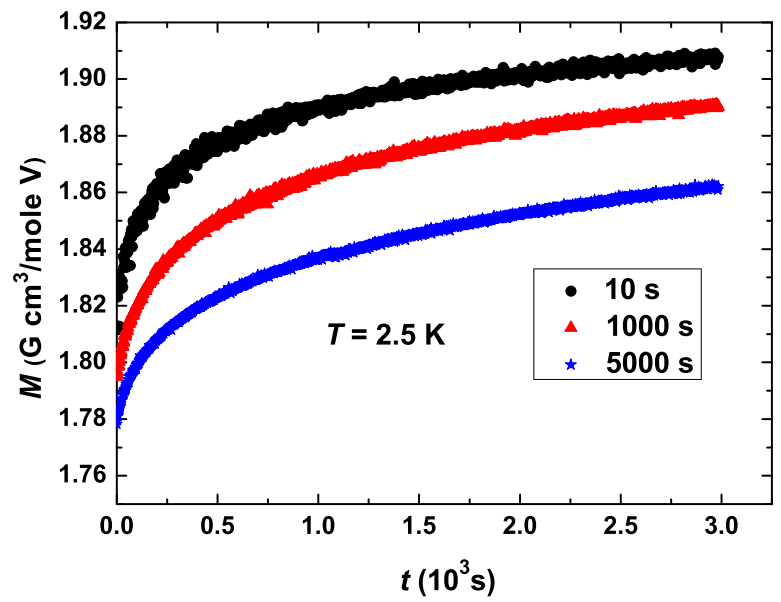

Figure 12: Aging effect is manifested by the growth of the magnetization as a function of time. The sample was cooled (ZFC) to $2.5 \mathrm{~K}$ and the field (200 Oe) was applied after different time intervals of 5,1000 and $5000 \mathrm{~s}$.

for a waiting time $t_{w}=3 \mathrm{~h}$, in each case. During $t_{w}$, the field was switched off and the system was allowed to relax. After each stop and wait period, the FC process was resumed. The stops at $2.7 \mathrm{~K}$, and $2.2 \mathrm{~K}$ are evident in the obtained $M_{F C C}^{S t o p}$ curve in Fig. 13, as step like features. Once the cooling process was completed by reaching $2 \mathrm{~K}$, the sample was heated continuously in the same magnetic field and heating rate while recording the magnetization data. The magnetization obtained this way, referred to as $M_{F C W}^{M e m}$, exhibits a weak change of slope at $2.7 \mathrm{~K}$ and a prominent minimum at $2.2 \mathrm{~K}$ although there is no stop at these temperatures. This in- 


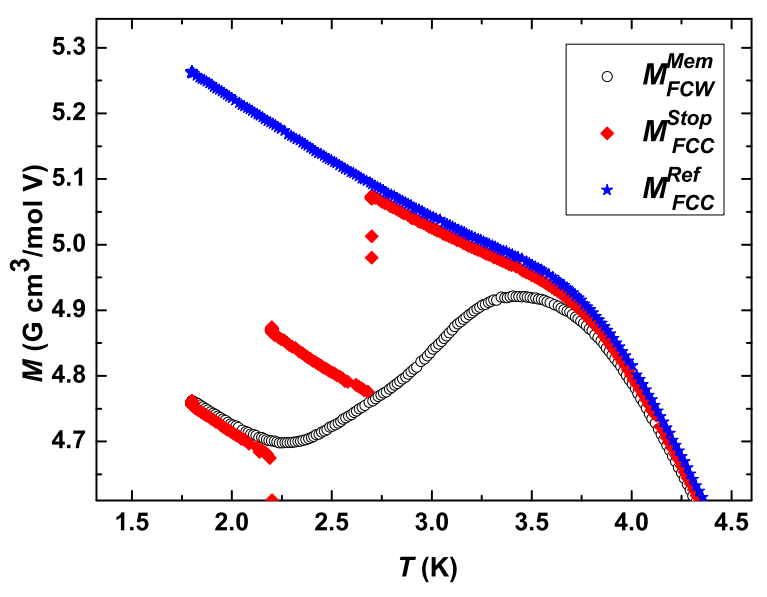

Figure 13: The memory effect in $\mathrm{Zn}_{3} \mathrm{~V}_{3} \mathrm{O}_{8}$ is observed in the temperature variation of FC magnetization. Here $M_{F C C}^{\text {Stop }}$ curve was obtained during cooling the sample in a field of 500 Oe with intermediate stops of $3 \mathrm{~h}$ duration each at $2.7 \mathrm{~K}$ and $2.2 \mathrm{~K}$. At each stopping temperature, the field was switched off. $M_{F C W}^{M e m}$ was measured during continuous heating of the sample in the same field. The reference curve $\left(M_{F C C}^{R e f}\right)$ was measured during continous cooling of the sample in the same field $(H=500$ Oe).

dicates that the system has its previous behavior during the cooling operation imprinted as a memory. This sort of behavior has been observed in intermetallic compounds such as $\mathrm{GdCu}\left(\operatorname{Ref} . \frac{5}{*}\right), \mathrm{Nd}_{5} \mathrm{Ge}_{3}\left(\operatorname{Ref}^{6} \cdot \underline{6}\right)$ and in superspin glass nanoparticle systems $\frac{16,36}{}$ This is considered to be a typical characteristic of spin glasses. The dip at $2.7 \mathrm{~K}$ in the $M_{F C W}^{M e m}$ curve is weak because at $2.7 \mathrm{~K}$ the system is not much below the blocking temperature $\left(T_{b}=3.8\right.$ $\mathrm{K}$ at $H=500 \mathrm{Oe}$ ) which is the peak of the ZFC curve (see Fig. 3). This signifies that at $2.7 \mathrm{~K}$ the system is not deep enough into the SG state. A reference curve $\left(M_{F C W}^{R e f}\right)$ was also measured by simply cooling the sample continuously in $H=500$ Oe. No memory effect has been observed when we waited at $15 \mathrm{~K}$, a temperature above $T_{b}$ (not shown in Fig. 13).

To further test the signature of the memory effect we have investigated the ZFC and FC relaxation behavior with negative $T$ cycling as shown in Fig. 14] In the ZFC method, the sample was first zero field cooled down from the paramagnetic phase to the measuring temperature $T_{1}=3.2 \mathrm{~K}$, which is below the spin freezing temperature $T_{f}$. Subsequently, a magnetic field of 500 Oe was applied and the magnetization was recorded as a function of time for a time period $t_{1}=1 \mathrm{~h}$. After that, the sample was quenched to a lower temperature $T_{2}=1.9$ $\mathrm{K}$ without changing the field and the magnetization was recorded for a time $t_{2}=1 \mathrm{~h}$. Finally, the temperature was restored to $T_{1}=3.2 \mathrm{~K}$ and the magnetization was recorded for a time $t_{3}=1 \mathrm{~h}$. The relaxation curve
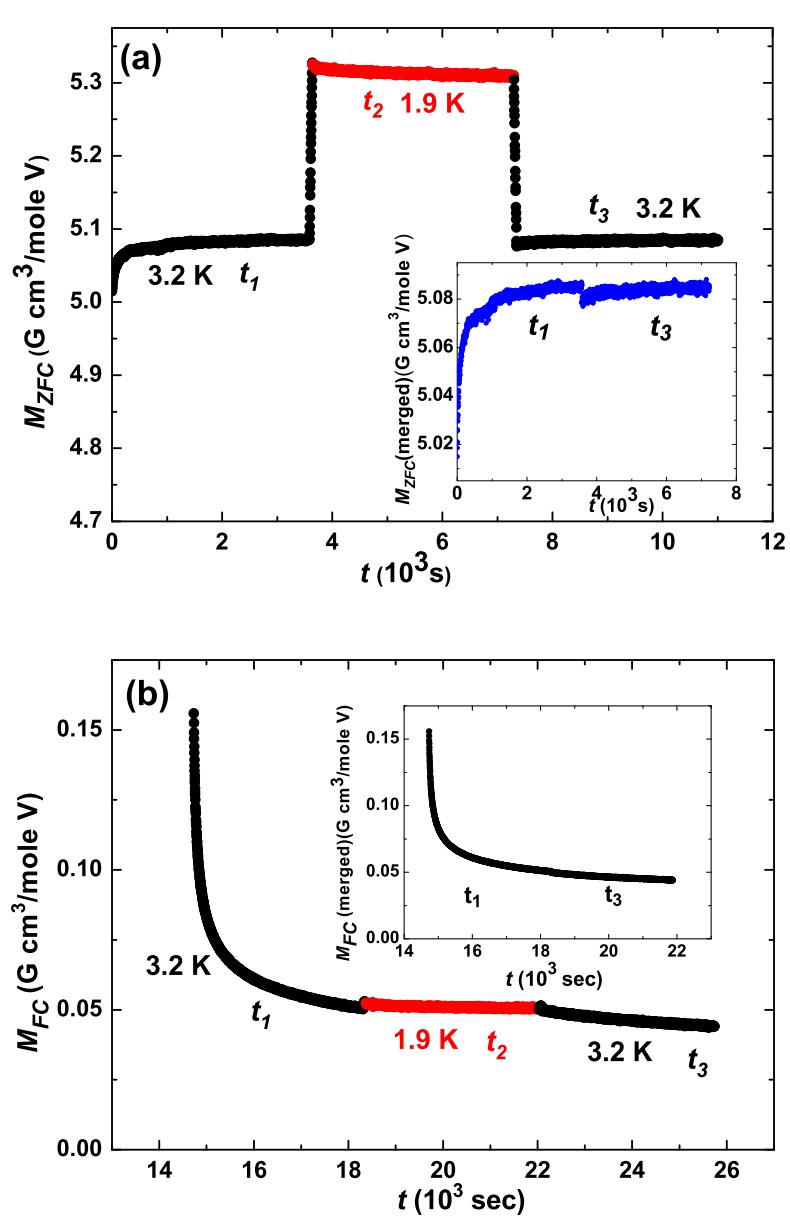

Figure 14: Magnetic relaxation in $\mathrm{Zn}_{3} \mathrm{~V}_{3} \mathrm{O}_{8}$ at $3.2 \mathrm{~K}$ with a temporary quench to $1.9 \mathrm{~K}$ for (a) the ZFC method and (b) the FC method. Insets show the relaxation data during $t_{1}$ and $t_{3}$ merge nearly perfectly.

obtained this way is depicted in Fig. 14(a). When the system was returned to $T_{1}=3.2 \mathrm{~K}$ after the temporary quenching, the magnetization resumes from the previous value it reached before the temporary quenching. This indicates that the temporary quenching does not erase the memory in ZFC relaxation. In the FC process, the sample was first field cooled to $T_{1}=3.2 \mathrm{~K}$ in 500 Oe. Once the measuring temperature was reached, the field was switched off and subsequently the magnetization was measured as a function of time (see Fig. 14(b)). Similar to the ZFC method, the FC method also preserves the state of the system even after a temperature quench. In both ZFC and FC methods, the relaxation curve during $t_{3}$ is just a continuation of the curve during $t_{1}$ as shown in the insets of Fig. 14, which represents a memory effect.

According to the droplet mode ${ }^{37,38}$ of spin glasses, one would expect a symmetric behavior in magnetic relaxation with respect to heating and cooling. But the hierarchical mode $\mathbb{1 6}^{16,17}$ predicts that a positive temperature cycle can destroy the previous memory and initialize the 
relaxation again, which means the response is asymmetric. In order to compare the response with respect to intermittent heating and cooling cycles, the relaxation experiment with a temporary heating cycle was also performed. The results are shown in Fig. 15(a) and (b). As can be seen from these figures, a positive temperature cycling erases the memory and reinitializes the relaxation in both ZFC and FC processes. This clearly suggests that the response of the system is asymmetric, therefore it supports the hierarchical picture proposed for spin glasses. According to this model, there exists a multi-valley free-energy surface of a frustrated system at a given temperature $T$. When the temperature of the system is lowered from $T$ to $T-\Delta T$, then each valley is split into many sub-valleys. If $\Delta T$ is large the energy gaps between the primary valleys are also large, and the system cannot overcome this energy barrier within a given time $t_{2}$. So, the relaxation occurs only within the secondary sub-valleys. When the system is brought back to its intial temperature temperature $T$, the sub-valleys coalesce back to the original free-energy surface and relaxation at $T$ resumes without being perturbed by the intermediate relaxations at $T-\Delta T$. But when the system is heated from $T$ to $T+\Delta T$, then the barriers between the free energy primary valleys are lowered or sometimes even merge. Therefore, the relaxations can easily take place within different primary valleys. When the temperature $T$ is restored, although the free energy surface goes back to the original free energy surface, the relative occupancy of each primary energy valley does not remain the same as before. Therefore, the state of the system changes after a temporary heating cycle and no memory effect is observed. Behavior of the kind observed in $\mathrm{Zn}_{3} \mathrm{~V}_{3} \mathrm{O}_{8}$ has been seen in some other SG systems too $\underline{5,6,16}$

\section{B. Heat capacity}

The heat capacity data of $\mathrm{Zn}_{3} \mathrm{~V}_{3} \mathrm{O}_{8}$ are shown in Fig. 16. It is clear from the figure that no sign of long-range ordering is observed down to $2 \mathrm{~K}$. A small hump is seen in the $C_{\mathrm{p}} / T$ data, at around $3.75 \mathrm{~K}$, which shifts slightly with the increase in the magnetic field. However this change with magnetic field is not due to the Schottky anomaly.

Since we do not have a suitable non-magnetic analogue for this system we tried to extract the magnetic specific heat of $\mathrm{Zn}_{3} \mathrm{~V}_{3} \mathrm{O}_{8}$ by subtracting the lattice contribution using a combination of Debye and Einstein heat capacities, $C_{\text {Debye }}$ and $C_{\text {Einstein }}$, respectively. In the $T$-range $30-110 \mathrm{~K}$, the measured heat capacity $C_{P}$ could be fit with a combination of one Debye and two Einstein functions of the type given below where the coefficient $C_{d}$ stands for the relative weight of the acoustic modes of vibration and the coefficients $C_{e_{1}}$ and $C_{e_{2}}$ are the relative weights of the optical modes of vibration.

$$
C_{\text {Debye }}=C_{d} \times 9 n R\left(T / \theta_{\mathrm{d}}\right)^{3} \int_{0}^{\theta_{\mathrm{d}} / T}\left(x^{4} e^{x} /\left(e^{x}-1\right)^{2}\right) d x
$$
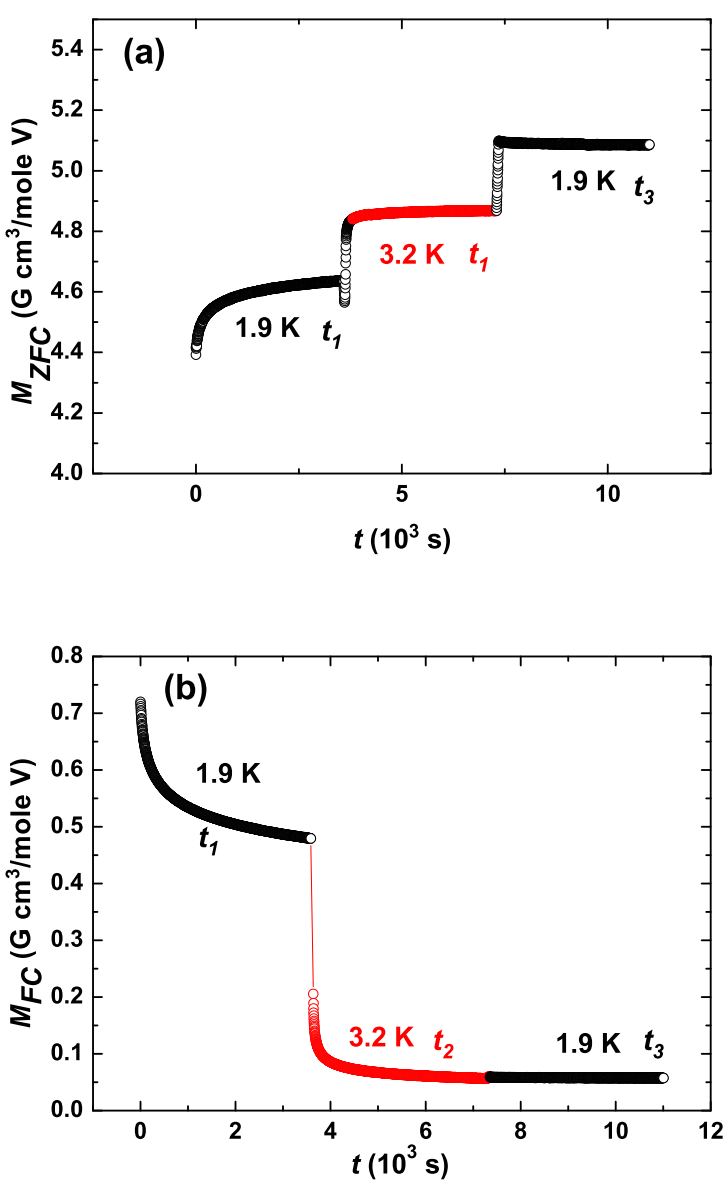

Figure 15: Magnetic relaxation in $\mathrm{Zn}_{3} \mathrm{~V}_{3} \mathrm{O}_{8}$ with a positive heating cycle for (a) ZFC method and (b) FC method.

$$
C_{\text {Einstein }}=3 n R\left[\sum C_{e_{m}} \times \frac{x_{E_{m}}^{2} e^{x} E_{m}}{\left(e^{x} E_{m}-1\right)^{2}}\right], x=\frac{h \omega_{E}}{k_{B} T}
$$

In the above formula, $n$ is the number of atoms in the primitive cell, $k_{\mathrm{B}}$ is the Boltzmann constant, and $\theta_{\mathrm{d}}$ is the relevant Debye temperature, $m$ is an index for an optical mode of vibration. In the Debye-Einstein model the total number of modes of vibration (acoustic plus optical) is equal to the total number of atoms in the primitive unit cell. For $\mathrm{Zn}_{3} \mathrm{~V}_{3} \mathrm{O}_{8}$ this number is 14 . In this model we have considered the ratio of the relative weights of acoustic modes and sum of the different optical modes to be $1: n-1$. Due to having two heavy atoms (vanadium and zinc) and one comparatively lighter atom (oxygen) in this compound we considered two different optical modes of vibrations. The fit yields a Debye temperature of $127 \mathrm{~K}$ and Einstein tempertures of $249 \mathrm{~K}$ and $600 \mathrm{~K}$ with relative weights $C_{d}: C_{e_{1}}: C_{e_{2}}=2.6: 12: 19$. Upon subtracting the lattice heat capacity with the above parameters, we obtain the magnetic contribution to the heat capacity $C_{\mathrm{m}}(T)$. The entropy change $(\Delta S)$ was calculated by integrating the $C_{m} / T$ data (see Fig. 16). The entropy change from about $14 \mathrm{~K}$ to $2 \mathrm{~K}$ is about $1.76 \mathrm{~J} / \mathrm{K}$ (cal- 


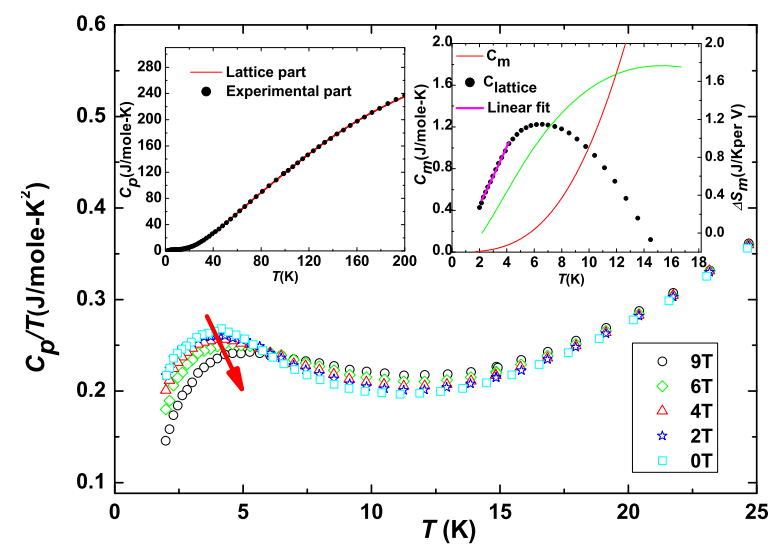

Figure 16: (Left inset) The temperature dependence of specific heat of $\mathrm{Zn}_{3} \mathrm{~V}_{3} \mathrm{O}_{8}$; the red line represents the fitting of the heat capacity using one Debye and two Einstein terms (see text). The right inset displays the magnetic contribution of specific heat at zero field, the red line indicates the lattice contribution of heat capacity, the magenta line shows the fit with the power law (given in text); the green line (right axis, left inset) shows the change of entropy calculated by integrating the $C_{\mathrm{m}} / T$ data. In the main figure $C_{P} / T$ vs. $T$ is shown at zero field (cyan square), $20 \mathrm{kOe}$ (blue star), $40 \mathrm{kOe}$ (red triangle), $60 \mathrm{kOe}$ (green diamond) and $90 \mathrm{kOe}$ (black circle) to depict the slight shift of the maximum of $C_{P} / T$ curve with the increase in magnetic field. Note that in both the insets and in the main figure the heat capacity is calculated for one formula unit.

culated for one formula unit containing three vanadium ions). For $S=1 / 2$ and $S=1$ systems the values of the entropy change $(\Delta S=R \ln (2 S+1))$ are $5.763 \mathrm{~J} / \mathrm{K}$ and $9.314 \mathrm{~J} / \mathrm{K}$ respectively. If two vanadiums in $\mathrm{Zn}_{3} \mathrm{~V}_{3} \mathrm{O}_{8}$ are in the $3+$ oxidation state $(S=1)$ and the remaining one is in the $4+$ state $(S=1 / 2)$ then the total entropy change expected in case of long range order would be $24.391 \mathrm{~J} / \mathrm{K}$. The value of $\Delta S$ obtained for $\mathrm{Zn}_{3} \mathrm{~V}_{3} \mathrm{O}_{8}$ is only about $7 \%$ of this value which indicates the presence of many degenerate low-energy states at low temperatures ${ }^{39}$ This large reduction in the value of $\Delta S$ down to temperatures much lower than the Weiss temperature $\left(\theta_{C W}\right)$ is typical of disordered systems and a consequence of the presence of strong geometric frustration in $\mathrm{Zn}_{3} \mathrm{~V}_{3} \mathrm{O}_{8}$. We observed a broad maximum at $6 \mathrm{~K}$ in the $C_{\mathrm{m}}(T)$ vs. $T$ data above the freezing temperature $\left(T_{f}\right)$ which is a characteristic feature of spin-glass systems $\frac{25,40}{2}$ At low $T$, (in the range of $2-4 \mathrm{~K}) C_{\mathrm{m}}(T)$ varies nearly as $C_{\mathrm{m}}(T)=\gamma T^{\alpha}$ with $\gamma=0.21 \mathrm{JK}^{-2.2} \mathrm{~mol}^{-1}$ and $\alpha=1.15$, i.e., nearly linear with temperature. Particularly in SG systems this kind of linear variation of the low-temperature magnetic specific heat is claimed to be a common feature $\underline{\underline{41}} \underline{\underline{43}}$. All these facts collectively point towards the formation of a metastable frozen state in the system at low temperature. IV. CONCLUSION

In this work we have reported the crystal structure, $d c$ and $a c$ magnetization, various static and dynamic magnetic measurements, and heat capacity of a new vanadium-based multivalenced spinel $\left(\mathrm{AB}_{2} \mathrm{O}_{4}\right)$ system $\mathrm{Zn}_{3} \mathrm{~V}_{3} \mathrm{O}_{8}$. The $\chi(T)$ data evidence the presence of strong antiferromagnetic correlations between the magnetic ions. The Curie constant is consistent with the presence of two vanadiums in the $3+$ oxidation state and one in the $4+$ state in each formula unit. Below about 3.75 $\mathrm{K}$ (well below $\theta_{C W}$ of $-370 \mathrm{~K}$ ), ZFC and FC magnetization curves bifurcate from each other which is suggestive of spin glass behaviour. This is supported by the measurements of $a c$ susceptibility where we observed a peak at about $3.75 \mathrm{~K}$ (both in $\chi^{\prime}$ and $\chi^{\prime \prime}$ ) and the freezing temperature has a logarithmic variation with the change in the measuring frequency. Below $T_{g}, \chi^{\prime \prime}$ attains a constant value which again provides a signature of $\mathrm{SG}$ behavior in $\mathrm{Zn}_{3} \mathrm{~V}_{3} \mathrm{O}_{8}$. From the value of the characteristic frequency $\left(\omega_{0}=0.29 \times 10^{5} \mathrm{rad} / \mathrm{s}\right)$ obtained from the Vogel-Fulcher fit, we conclude that the system is closer to a cluster spin glass where the magnetic entities involved are bigger than at the atomic level. We also observed the relaxation of isothermal remanent magnetization and the growth of the ZFC magnetization, which are very commonly observed in SG systems. Further, ageing phenomena and memory effect were also observed both in ZFC and FC magnetization. We observed that a positive temperature cycle erases the memory while a negative temperature cycle retains memory. This type of behavior is predicted by the hierarchical model of SG systems. In the heat capacity measurement we did not observe any sharp anomaly indicative of long range ordering down to $2 \mathrm{~K}$. At around $3.75 \mathrm{~K}$ a small hump is seen in the $C_{\mathrm{p}} / T$ data. The entropy change $\Delta S$ is only about $7 \%$ of what is expected for an ordered system containing two $S=1$ and one $S=1 / 2$ ions per formula unit. This is likely due to the presence of strong geometric frustration in the system. The magnetic heat capacity shows an almost linear variation below $T_{f}$, which is another common feature in SG systems. All these above features point to the formation of a metastable, nonergodic state in $\mathrm{Zn}_{3} \mathrm{~V}_{3} \mathrm{O}_{8}$ below 3.75 K.

\section{ACKNOWLEDGEMENT}

Discussions with B. Koteswararao are acknowledged. The authors thank the Department of Science and Technology, Govt. of India for financial support. 
1 Greedan J E 2001 J. Mater. Chem. 1137

2 Moessner R and Ramirez A P 2006 Physics Today 5924

3 Binder K and Young A P 1986 Rev. Mod. Phys. 58801

4 Jonason K, Vincent E, Hammann J, Bouchaud J P and Nordblad P 1998 Phys. Rev. Lett. 813243

5 Bhattacharyya A, Giri S and Majumdar S 2011Phys. Rev. B 83134427

6 Maji B, Suresh K G and Nigam A K 2011 J. Phys.: Condens. Matter 23506002

7 Deac G, Mitchell J F and Schiffer P 2001 Phys. Rev. B 63 172408

8 Maignan A, Martin C, Damay F, Raveau B and Hejtmanek J 1998 Phys. Rev. B 582758

9 Malinowski A, Bezusyy V L, Minikayev R, Dziawa P, Syryanyy Y and Sawicki M 2011 Phys. Rev. B 84024409

10 Tang Y, Sun Y and Cheng Z 2006 Phys. Rev. B 73012409

11 Dho J, Kim W S and Hur N H 2001 Phys. Rev. B 65 024404

12 Roy S B, Chattopadhyay M K, Chaddah P, Moore J D, Perkins G K, Cohen L F, Gschneidner K A Jr and Pecharsky V K 2006 Phys. Rev. B 74012403

13 Haldar A, Suresh K G and Nigam A K 2010 Eur. Phys. Lett. 9167006

14 Viswanathan M and Anil Kumar P S 2009 Phys. Rev. B 80012410

15 Sampathkumaran E V and Niazi A 2002 Phys. Rev. B 65 180401

16 Sun Y, Salamon M B, Garnier K and Averback R S 2003 Phys. Rev. Lett. 91167206

17 Lefloch F, Hammann J, Ocio M and Vincent E 1992 Eur. Phys. Lett. 18647

18 Tachibana M, T Tojo, Hitoshi K, Tooru A, Hiromasa I, Yoshiharu U and Masataka W 2002 Phys. Rev. B 66 092406

19 Selwood P W 1956 Magnetochemistry (Interscience New York )

20 Derakhshan S, Greedan J E and Cranswick L M D 2008 Phys. Rev. B 77014408

${ }^{21}$ Li D X, Nimori S, Shiokawa Y, Tobo A, Onodera H, Haga Y, Yamamoto E and Onuki Y 2001 Applied Physics Letters 794183

${ }^{22}$ Li D X, Nimori S, Shiokawa Y, Haga Y, Yamamoto E and Onuki Y 2003 Phys. Rev. B 68172405

${ }^{23}$ Kuboinowski A M, Bezusyy V L, Minikayev R, Dziawa P, Syryanyy Y and Sawicki M 2011 Phys. Rev. B 84024409

24 Mulder C A M, Van Duyneveldt A J and Mydosh J A 1982
Phys. Rev. B 25515

25 Süllow S, Nieuwenhuys G J, Menovsky A A, Mydosh J A, Mentink S A M, Mason T E and Buyers W J L 1997 Phys. Rev. Lett. $\mathbf{7 8} 354$

${ }^{26}$ Fisher D S and Huse D A 1986 Phys. Rev. Lett. 561601

27 Giot M, Pautrat A, André G, Saurel D, Hervieu M and Rodriguez-Carvajal J 2008 Phys. Rev. B 77134445

28 Mydosh J A 1993 Spin Glasses: An Experimental Introduction (Taylor \& Francis, London )

${ }^{29}$ Nam D N H, Mathieu R, Nordblad P, Khiem N V and Phuc N X 2000 Phys. Rev. B 628989

30 Singh M K, Prellier W, Singh M P, Katiyar R S and Scott J F 2008 Phys. Rev. B $\mathbf{7 7} 144403$

31 De K, Thakur M, Manna A and Giri S 2006 J. Appl. Phys. 99013908

32 Mulder C A M, Van Duyneveldt A J and Mydosh J A 1981 Phys. Rev. B 231384

33 Chatterjee S, Giri S, De S K and Majumdar S 2009 Phys. Rev. B 79092410

${ }^{34}$ Li D X, Shiokawa Y, Homma Y, Uesawa A, Dönni A, Suzuki T, Haga Y, Yamamoto E, Honma T and Onuki Y 1998 Phys. Rev. B 577434

35 Mathieu R, Jönsson P, Nam D N H and Nordblad P 2001 Phys. Rev. B 63092401

36 Sasaki M, Jönsson P E and Takayama H 2005 Phys. Rev. B 71104405

37 Fisher D S and Huse D A 1988 Phys. Rev. B 38373

38 Fisher D S and Huse D A 1988 Phys. Rev. B 38386

39 Depending on the temperature range chosen for the fit, the inferred lattice heat capacity is a bit different. Also, since this system has a large $\theta_{C W}(=-370 \mathrm{~K})$, entropy release might take place in a broad temperature range. Due to the large lattice contribution at high temperatures it is nearly impossible to accurately determine the magnetic contribution to the heat capacity in this range. Consequently, there is likely to be a significant uncertainty in the entropy change estimation.

40 Brando M, B“ uttgen N, Fritsch V, Hemberger J, Kaps H, Krug von Nidda H-A, Nicklas M, Pucher K, Trinkl W, Loidl A, Scheidt E W, Klemm M and Horn S 2002 Eur. Phys. J. B 25289

41 Wenger L E and Keesom P H 1975 Phys. Rev. B 113497

42 Wenger L E and Keesom P H 1976 Phys. Rev. B 134053

43 Meschede D, Steglich F, Felsch W, Maletta H and Zinn W 1980 Phys. Rev. Lett. 44102 